Apothekerschaft und GKV-

\section{Spitzenverband verhandeln}

Dem Ersatzkassenverband vdek hat die ABDA nach Angaben von Sprecher Christian Splett immerhin die Zusage abgetrotzt, bis Ende September Rezepte nicht zu retaxieren, auf denen Telefon und Arztvorname fehlen. Was im Umkehrschluss aber auch bedeutet, dass dies in absehbarer Zeit eben doch einen Anlass für Retaxationen geben könnte. Eine „endgültige Lösung“, so Splett, sei noch auszuhandeln.

Neben den Ersatzkassen und der AOK Rheinland-Hamburg wird auch die Knappschaft zunächst auf eine Rechnungskürzung verzichten, wenn Rezepte nach der Umstellung der Vorgaben zum 1. Juli nicht den neuesten Anforderungen entsprechen. Wie die Krankenkasse berichtet, wird sie eine dreimonatige Übergangsfrist gewähren, „da die Umstellung der Rezeptvordrucke noch nicht in allen Arztpraxen abgeschlossen ist".

Und wie verfährt der Apotheker nun mit Rezepten, die unvollständig ausgefüllt sind? Eigenhändig darf er laut AMVV nur ganz bestimmte Angaben auf dem Rezept ergänzen. Und das auch nur dann, „wenn ein dringender Fall vorliegt und eine Rücksprache mit der verschreibenden Person nicht möglich ist". Telefonnummer und Arztvorname gehören nicht dazu. Auch die etwa im Versorgungsvertrag mit den Ersatzkas- sen vorgesehene Möglichkeit, dass Apotheker fehlende Rezeptangaben selbst nachtragen, scheidet aus, solange der Vertrag nicht um eine entsprechende Erwähnung der neuen Formerfordernisse ergänzt wird.

Apotheker müssen Rezepte ohne Praxis-Telefonnummer und Arztvorname also zurück schicken. „Erhält der Arzt eine unvollständige Verordnung zurück, reicht es aus, wenn er die fehlenden Angaben handschriftlich ergänzt. Eine erneute Unterschrift ist nicht erforderlich", lautet jedenfalls auf Anfrage die Rechtsauffassung der ABDA. „Eine erneute Unterschrift wäre nur dann erforderlich, wenn die verordnete Leistung geändert würde."

Christoph Winnat

\title{
Im Depot sind weiter Aktien angesagt
}

Größere Kursschwankungen an der Börse gehören heutzutage beinahe schon zum Alltagsgeschäft. Anleger macht das nicht minder nervös, dabei setzen Experten auf ein stabiles Wachstum für die deutsche Wirtschaft.

$\mathrm{N}$ ach der starken Aufwärtsbewegung an der Börse im ersten Halbjahr hat es in den vergangenen Monaten starke Kursschwankungen gegeben. Auslöser waren zunächst die Turbulenzen um einen möglichen Ausstieg Griechenlands aus dem Euro gewesen, aber auch die Schwächetendenzen an Chinas Börsen machten die Anleger nervös. Für Klaus Niedermeier, Abteilungsleiter Asset Management bei der apoBank, stehen dennoch angesichts weiterhin sehr niedriger Zinsen die Aktien im Vordergrund bei den Anlageoptionen. „Auch wenn die Lösung mit Griechenland noch keine dauerhafte ist: Die Unsicherheit ist zunächst einmal weg, und das ist positiv", so die Einschätzung Niedermeiers. Auch das Ende des Atomkonflikts mit dem Iran habe rein wirtschaftlich positive
Auswirkungen, der Anlageexperte sieht für 2016 ein zusätzliches Wachstumspotenzial von 0,25 Prozentpunkten für die deutsche Wirtschaft. Alle Frühindikatoren deuteten derzeit eher auf stabiles Wachstum in Deutschland hin.

\section{Anlagen breit streuen}

„Entscheidend ist es aber immer, die Anlagen breit zu streuen", betont Niedermeier. So könnten Marktschwankungen in einzelnen Regionen gut ausgeglichen werden. Er empfiehlt derzeit unter anderem eine leichte Übergewichtung der Märkte in Europa, ausdrücklich auch in der Peripherie: „In Italien und Spanien zum Beispiel gibt es große Aufholpotenziale“, so Niedermeier. In Asien seien nach den Problemen in China derzeit andere Zugpferde gefragt, etwa Indien oder auch Südkorea, nachdem die MersInfektion jetzt unter Kontrolle gebracht worden ist. Insgesamt bleibe die Wachstumsgeschwindigkeit in dieser Region aber höher als in Europa oder anderen Regionen der Welt. In den USA sei vor dem ersten Zinsschritt nach oben - er steht möglicherweise im September an zunächst Vorsicht angesagt.

Um bei Anleihen Renditen erwirtschaften zu können, ist es nach Meinung des Experten sinnvoll, zum Teil ins Risiko zu gehen, etwa über Unternehmensanleihen. „Aber man sollte auf keinen Fall zu viel Geld auf eine Karte setzen", warnt Niedermeier. Nicht bei jedem Unternehmen und in jeder Region müssen sich Anleger jedoch auskennen, wenn sie investieren. Niedermeier empfiehlt, auf breit gestreute Dachfonds zu setzen, die sowohl Anleihen als auch Aktien im Portfolio haben. Je nach Risikoscheu könnten Anleger auf offensiv ausgerichtete Fonds (hohe Aktienquote), ausgewogene oder defensiv ausgerichtete Fonds (niedrige Aktienquote) setzen.

Hauke Gerlof

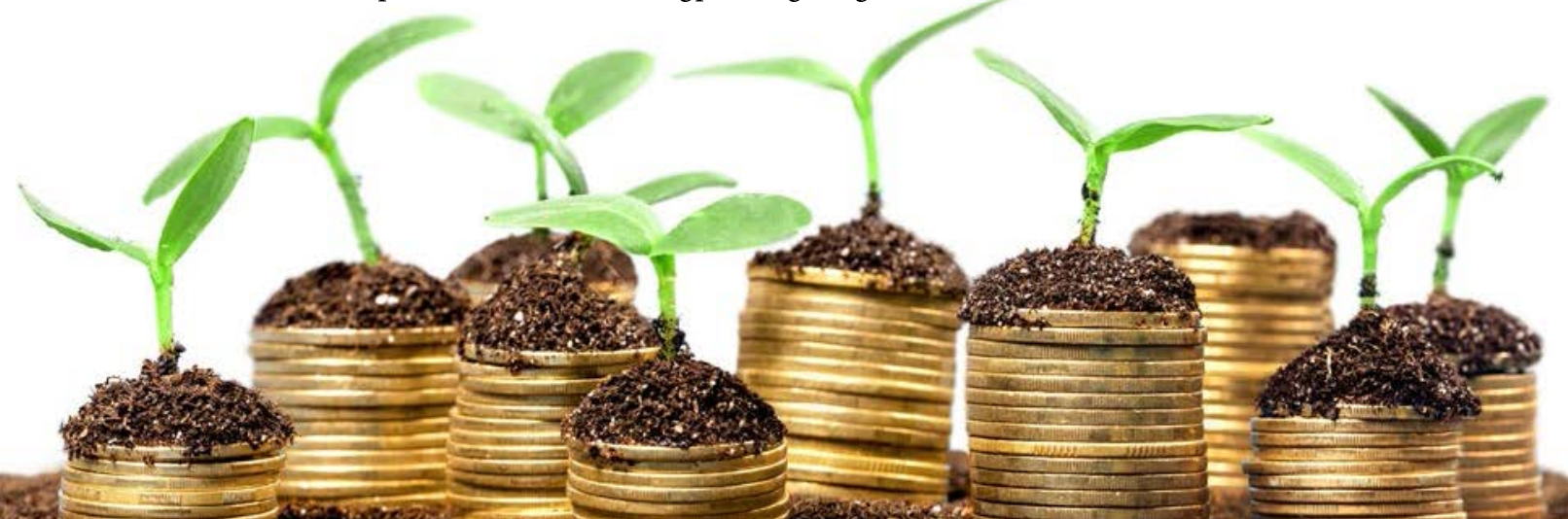

